The assembly process was achieved by dissolving the diphenylalanine building blocks in a fluorinated solvent, 1,1,1,3,3,3hexafluoro-2-propanol (HFP). In this solvent, the peptides do not interact with one another and are easily dissolved. However, HFP is very volatile and rapidly evaporates, so the peptides are deposited from solution on to a surface. Gazit and Reches suggest that this process forms numerous sites from which nanotubes grow in a unidirectional fashion. Highly aligned nanotube forests were formed across the entire surface of the glass substrate on which the HFP solution was dropped. However, an alternative assembly mechanism, in which complete nanotubes first assemble in the bulk solution and then arrange themselves on to the surface to form a forest-like structure, cannot be ruled out.

Although the precise pathway of the growth is not fully understood at this stage, these findings pave the way to grow, molecule by molecule, well-structured nanotubes that may be useful for a wide range of applications. For example, it was previously reported that individual dipeptide nanotubes could be used as templates, inside which metal nanowires could be grown ${ }^{8}$. Furthermore, dense nanotube arrays with large surface areas and the capability to interact with other biological molecules could lead to highsensitivity sensors for both environmental and medical diagnostic applications.

Gazit and Reches also show how these peptide nanotubes can be aligned horizontally. The high-yielding assembly of peptide nanotubes can be performed in the presence of magnetic particles, which end up coating the outside of the tubes. These magnetically modified structures were spread on to a surface where they adopted random orientations. However, the nanotubes were seen to realign in response to an applied field, resulting in both spatial organization and alignment. Altogether, Gazit and Reches have demonstrated how to control both the vertical and horizontal arrangement of self-assembled peptide nanotubes using quite simple, yet elegant, methods.

What makes this study especially interesting is the simplicity with which the well-defined nanotubes can be made. Although there have been reports concerning the production of nanotubes using other materials, this example uses a small, yet biologically relevant system, to grow large arrays of well-aligned highintegrity tubes. It is remarkable that such a basic building block can assemble into highly regular forest-like structures. Not only from small acorns do large trees grow, so do entire forests! It will be interesting to see if other aromatic dipeptides, either natural or man-made, can self-assemble into ordered nanostructures.

The authors' method is not just simple, but relatively inexpensive. As the basic starting materials are all commercially available and the methods are straightforward, this technology could be adopted widely and quickly. If nanotechnology is to lead to new devices and products, the development of the simplest methods is one of the most important factors. It must be emphasized, however, that going from milligrams of material to kilograms or tonnes is not easy and challenges still remain. New innovative approaches for the chemical synthesis of peptides or perhaps even cellbased production may play an increasingly important role for wider applications of selfassembling peptide materials.

Although nature can build numerous elegant structures and molecular machines, it is up to us to construct technological devices. We are now learning from nature and trying to do even better. As an old Chinese proverb says, "dark blue (qing) is derived from blue (lan) but is even better than blue". It is believed that these simple and versatile selfassembling peptides will provide us with new opportunities not only to study complex and previously intractable biological phenomena, but also to make novel materials and intricate molecular machines.

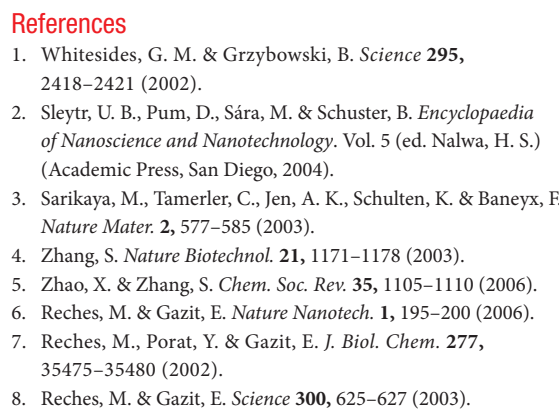

\section{References}

Whitesides, G. M. \& G

(Academic Press, San Diego, 2004).

Sarikaya, M. Tamerler, C. Jen, A.K. Schulten, K. \& Baneyx,

2. Zhao X. \& Z Zhang S. Chem. Soc. Rev. 35, 1105-1110 (2006).

. Reches, M. \& Gazit, E. Nature Nanotech. 1, 195-200 (2006) 35475-35480 (2002)

8. Reches, M. \& Gazit, E. Science 300, 625-627 (2003)

\title{
Surface patterning: Fullerenes line up
}

The ability to create arrays of organic nanostructures with long-range order and uniform size could have applications in electronic and optoelectronic devices. One of the most promising methods for making such arrays is to rely on the self-assembly of molecules on surfaces that already possess long-range order. Researchers at the Swiss Federal Laboratories for Materials Testing and Research in Thun and Liverpool University in the UK have now used this approach to grow ordered arrays of fullerene nanochains on a gold surface, as this image shows.

Roman Fasel and coworkers sublimated the fullerene molecules onto specially prepared gold substrates that naturally form a rectangular superlattice

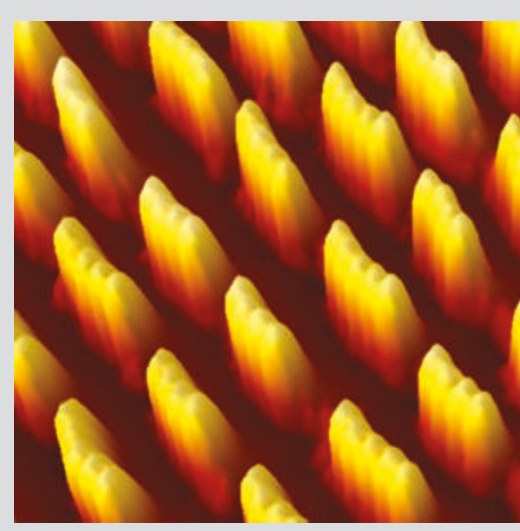

(J. Phys. Chem. B 110, $21394-$ 21398; 2006). Scanning tunnelling microscopy and low-energy electron diffraction revealed that the nanochains - which each contain just four or five fullerene molecules - only formed at the lower edges of the 'steps' that are found on the gold
Gold surfaces are natural templates for growing nanostructures.

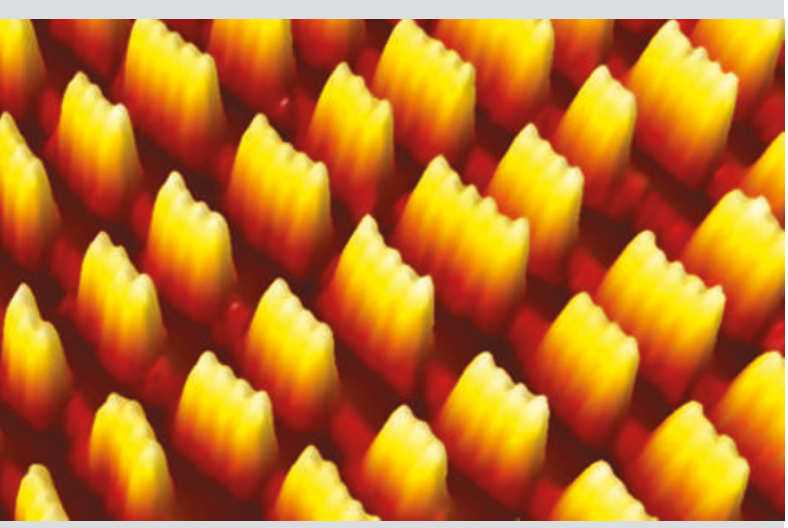

surface. It is thought that the electron-rich regions near these edges preferentially adsorb the fullerene molecules, which are electron acceptors, leading to the formation of arrays that perfectly reproduce the periodicity of the gold template.

Ai Lin Chun 